\title{
A strategy analysis for genetic association studies with known inbreeding
}

\author{
Stefano Cabras ${ }^{1 *}$, Maria Eugenia Castellanos ${ }^{2}$, Ginevra Biino ${ }^{5}$, Ivana Persico ${ }^{3}$, Alessandro Sassu ${ }^{4}$, Laura Casula ${ }^{3}$, \\ Stefano del Giacco ${ }^{6}$, Francesco Bertolino ${ }^{1}$, Mario Pirastu ${ }^{3,4}$ and Nicola Pirastu ${ }^{4,7}$
}

\begin{abstract}
Background: Association studies consist in identifying the genetic variants which are related to a specific disease through the use of statistical multiple hypothesis testing or segregation analysis in pedigrees. This type of studies has been very successful in the case of Mendelian monogenic disorders while it has been less successful in identifying genetic variants related to complex diseases where the insurgence depends on the interactions between different genes and the environment. The current technology allows to genotype more than a million of markers and this number has been rapidly increasing in the last years with the imputation based on templates sets and whole genome sequencing. This type of data introduces a great amount of noise in the statistical analysis and usually requires a great number of samples. Current methods seldom take into account gene-gene and gene-environment interactions which are fundamental especially in complex diseases. In this paper we propose to use a non-parametric additive model to detect the genetic variants related to diseases which accounts for interactions of unknown order. Although this is not new to the current literature, we show that in an isolated population, where the most related subjects share also most of their genetic code, the use of additive models may be improved if the available genealogical tree is taken into account. Specifically, we form a sample of cases and controls with the highest inbreeding by means of the Hungarian method, and estimate the set of genes/environmental variables, associated with the disease, by means of Random Forest.

Results: We have evidence, from statistical theory, simulations and two applications, that we build a suitable procedure to eliminate stratification between cases and controls and that it also has enough precision in identifying genetic variants responsible for a disease. This procedure has been successfully used for the betathalassemia, which is a well known Mendelian disease, and also to the common asthma where we have identified candidate genes that underlie to the susceptibility of the asthma. Some of such candidate genes have been also found related to common asthma in the current literature.

Conclusions: The data analysis approach, based on selecting the most related cases and controls along with the Random Forest model, is a powerful tool for detecting genetic variants associated to a disease in isolated populations. Moreover, this method provides also a prediction model that has accuracy in estimating the unknown disease status and that can be generally used to build kit tests for a wide class of Mendelian diseases.
\end{abstract}

\section{Background}

One of the main objectives in studying the genetics of complex diseases is not only the search of genetic variants associated to pathologies [1], but also to build predictive models which help both their diagnosis and early treatment. This problem can be formalized by expressing the disease status, $Y$, of each subject as a Bernoulli

\footnotetext{
* Correspondence: s.cabras@unica.it

'Department of Mathematics and Informatics, University of Cagliari, Cagliari, Italy

Full list of author information is available at the end of the article
}

random variable $Y=\{0,1\}$ where $Y=1$ indicates an affected subject. The main quantity of interest, $F(\mathbf{x})=\operatorname{Pr}$ $(Y=1 \mid \mathbf{x})$, is the conditional probability of being affected given a set $\mathbf{x}$ of genetic variants and environmental variables. Such variables form a huge set of potential predictors, which we will refer to as omic profile. Essentially, $\mathbf{x} \in \mathcal{X} \subset \mathrm{IR}^{P}$, where $P$ is the number of considered genetic variants and environmental variables. Specifically, we use a sample of $N \ll P$ where $N$ is of order of hundreds while $P$ is thousands times larger. This setup complicates the estimation of $F(\mathbf{x})$, because, in absence
Ciomed Central

() 2011 Cabras et al; licensee BioMed Central Ltd. This is an Open Access article distributed under the terms of the Creative Commons Attribution License (http://creativecommons.org/licenses/by/2.0), which permits unrestricted use, distribution, and reproduction in any medium, provided the original work is properly cited. 
of strong prior information [2] on the part of the omic profile related to the disease, the data should allow us to choose $F(\mathbf{x})$ within a large class of models $\mathcal{F}$. In order to achieve this goal it is necessary to reduce the spurious genetic variability not related to $Y$. For example, if we were using logistic regression models, $F(\mathbf{x})$ would be one of these models selected between all the possible logistic regression models, $\mathcal{F}$, which are, at least, $2^{P}$. In this paper we relax the assumption of a parametric model by using non-parametric methods [3], which means that $\mathcal{F}$ has infinite dimension. Such models are usually referred as non-parametric models. The genomic profile, part of the omic profile, consists of a large set of DNA markers, say 500000 Single-Nucleotide Polymorphisms (SNPs), while the set of environmental variables includes individual anthropometric measurements and information derived from a standardized interview collecting sociodemographic, lifestyle, medical and pharmacological history data on many pathologies. We suppose that such covariates may cause the outcome $Y$. In particular, for certain types of diseases, it is possible to have prior information about the environmental variables, but in most cases there is no such information about the causing genes. The disease prediction model, $F(\mathbf{x})$, for the future outcome $Y \mid \mathbf{x}$ must take into account gene-gene interactions and also their interaction with the environment. Such interactions, usually of unknown order, can be multiplicative or additive [4]. Estimation of $F(\mathbf{x})$ is a primary concern in personalized medicine, because $F(\mathbf{x})$ can be used as the basis for early diagnosis of a disease, permitting actions to prevent the pathology before its insurgence, and to personalize treatments.

In order to estimate $F(\mathbf{x})$, we consider a matrix of omic profiles, $\mathbf{X}_{N \times P}$, and the known disease status, $\mathbf{y}_{N}$ $=\left(y_{1}, y_{2}, \ldots, y_{N}\right)$, measured on $N \approx 100$ highly inbred individuals that belong to an isolate population where the genealogy is fully known.

The translation of this estimation problem into statistical terms sounds as follows: given a huge set of covariates, $P \approx 500000$, we have to estimate a probability model, $F(\mathbf{x}) \in \mathcal{F}$, using a sample of dependent observations, $\left(\mathbf{y}_{N}, \mathbf{X}_{N \times P}\right)$, of size $N \ll P$. The statistical analysis of such problem presents the following critical points:

i) observations are not independent and consequently all unconditional inference, with respect to the genealogy, cannot be applied here. Differently from usual association studies between genetic variants and diseases, we have knowledge of such dependency by means of the genealogical tree. Moreover, the dependency is important in order to gain precision in estimating $F(\mathbf{x})$. In fact, two affected brothers are more likely to share the same part of $\mathbf{x}$ causing the disease with respect to two unrelated subjects.

ii) the estimation of model $F(\mathbf{x})$ would typically lead to a sparse model because biological background suggests that only a very small set of genetic variants interact in order to produce the disease. The dimension of $\mathcal{F}$ grows exponentially with $P$. For example, if SNPs configurations were represented by categorical variables with two levels the space $\mathcal{F}$ would have dimension $2^{P}$, without considering interactions. Such dimension prevents an exhaustive exploration of all possible models. Moreover, as $N \ll P$ then classical multivariate analysis techniques, such as multivariate regression, cannot be employed here to make an exhaustive search of all possible models. Finally, usual model selection approaches are not feasible due to computational costs.

In this paper, we aim to address the above critical points. In particular, point $i$ ) is considered in the Methods Section by reducing the genetic variability not related with the disease. We achieve this through an experimental design in which we choose, for each case, the most related control, based on the known genealogy. Point ii) is treated also in the Methods Section where Random Forest, a non-parametric regression model based on ensemble methods, is employed to estimate $F(\mathbf{x})$. This allows us to explore a wide region of $\mathcal{F}$ at the price of reasonable computational costs.

For validation purposes, we present applications of the method to two different phenotypes: Beta-Thalassemia and common asthma. Beta-Thalassemia is a genetic disorder caused by a mutation inside the beta-hemoglobin (HBB) gene [5]. Only homozygous individuals for the mutation manifest the clinical traits of the disease. However, carriers, although completely sane, show a reduced mean cell volume $(\mathrm{MCV} \leq 72)$ of red blood cells [6], and this parameter can be used to identify them. In Sardinia carrier of beta-thalassaemia are about $15 \%$ of the population and a single mutation account for $95 \%$ of the beta-thalassaemia mutations $[7,8]$. The main goal of this analysis is to validate the method by tracing back the position of the mutation in the gene.

Differently from Beta-thalassemia, the goal of analyzing common asthma is to gain more biological insights on this diffuse disease which may be caused by several unknown variants on different genes.

Although the method here proposed is of general applicability to any isolated population, we tested it on a population located in one small village (Talana) in a secluded area (Ogliastra) of Sardinia (Italy). Such population is characterized by a great deal of homogeneity in life style and eating habits and by a high endogamy and consanguinity. Inhabitants of the village participated to 
an epidemiological survey assessing their health status, so that a complete and standardized data set is available. Thanks to the accessibility of complete municipal and parish archives, going back to the seventeenth century, it was possible to cluster all people living in the villages into large familiar structures with common ancestor. Data have been collected by Shardna Live Science http://www.shardna.com within the Ogliastra - project aimed at studying several genetic isolates of Ogliastra.

\section{Results and Discussion}

The following two sections present the application of the method here proposed to beta-Thalassemia and to common asthma.

\section{Application to Beta-Thalassemia}

One of the biggest issue in genome wide association studies is the number of false positive results due to the great number of association tests performed. In case-control studies it should be possible to reduce such number by choosing the control subjects so that they are the most genetically similar to the cases. We propose to apply the Hungarian algorithm $[9,10]$ to solve this problem by using the known kinship coefficient [11] as a measure of the genetic similarity between the subjects. The proposed approach also works if the kinship were estimated from the sample, which is however not the case here. The details on how the choice of the controls is done are described in the method section.

The aim of this application is to evaluate the power of the Hungarian method in choosing the best set of controls for a given sample of cases which correspond to all the Beta-Thalassemia mutation carriers in the isolated population we investigated. Such cases can be easily identified, among the available data, through the use of the MCV values measured for each subject in our study. In fact Beta-Thalassemia mutation carriers are known to show reduced $M C V$, namely we used $M C V \leq 72$ for cases and $M C V \geq 75$ for controls. With such cutoffs there are 123 cases, over a data set of 805 subjects. We then selected the subsample $\mathbf{D}$, where 123 controls have been chosen to be the most related to the $I=123$ cases, based on the kinship coefficient matrix of the 805 subjects. Analysis of such data is compared against other 9 balanced subsamples, of size $N=246$, where controls have been assigned randomly from the available 682 controls. Such assignation, that ignore the relativeness, is the usual one for observational studies from outbred populations.

Usual association studies are of single-point type and make use of hypothesis testing to evaluate the association between $\mathbf{y}_{N}$ and each SNP [12]. In order to evaluate if our subsample $\mathbf{D}$ would potentially provide a less number of false positives, due to the elimination of population stratification effect, we performed a genome wide scan on each of the 10 samples using Fisher exact test, that is, for each sample, we evaluate the evidence of the hypothesis of independence between $Y$ and each one of $P=429378$ SNPs. All tests are compared by means of their corresponding $p$-values. Figure 1 reports the most interesting part of the empirical cumulative distribution function (cdf) of the $p$-values less than 0.01 for each one of the ten considered subsamples. BetaThalassemia is a monogenic disease and therefore we expect a small proportion of SNPs associated with the $Y$ status. We can see that, when using $\mathbf{D}$, the proportion of p-values below 0.001 is smaller than the corresponding ones obtained with the other subsamples. This provides the first evidence of the reduction of the genetic variability operated by the introduction of genealogical information by means of the Hungarian method. We note that the rank of SNPs produced by the very simple Fisher test, used in the simulation study for Thalassemia, is the same as the one produced by the Benjamini Hochberg $(\mathrm{BH})$ method discussed in [13]. This is because ordered $p$-values are multiplied for the increasing sequence $i / P, i=1, \ldots, P$. As the BH method controls the False Discovery Rate (FDR) under the condition of positive dependence among tests discussed in [14], this implies that the rank of SNPs obtained for the Thalassemia is the same as those that we would have obtained also considering the dependency among tests. The analysis here performed can be also viewed as a kind of multi-point analysis. We further pursue this comparison by looking at the prediction errors, $\Lambda$, of the estimated Random Forest $(\mathcal{R} \mathcal{F})$ model, $\widehat{F}(\mathbf{x})$, based only on the most important SNP and its distance from the HBB gene. We have one important SNP for each data set and this SNP has been selected using the Bagging approach as detailed in the Methods Section. The Bagging approach is enough in this application because the Beta-Thalassemia is a monogenic disease. Prediction errors and distances, for all samples, are reported in Table 1 . We can see that only using sample $\mathbf{D}$, the prediction error is the lowest (12\%) and the selected SNP is also one of the nearest SNP to the HBB gene. Using other samples, we obtain larger prediction errors that often point to SNPs that are farther from the HBB gene.

Comparing $\mathcal{R} \mathcal{F}$ approach with the single-point analysis, carried out with Fisher test on subsample D, we obtain that SNP rs12271916 is the most significantly associated according to the Fisher test, but it is also located at $580 \mathrm{~Kb}$ from the $\mathrm{HBB}$ gene farther away from the SNP obtained with $\mathcal{R} \mathcal{F}$.

The final message conveyed by Figure 1 and Table 1 is that the subsample $\mathbf{D}$ has lower genetic spurious 


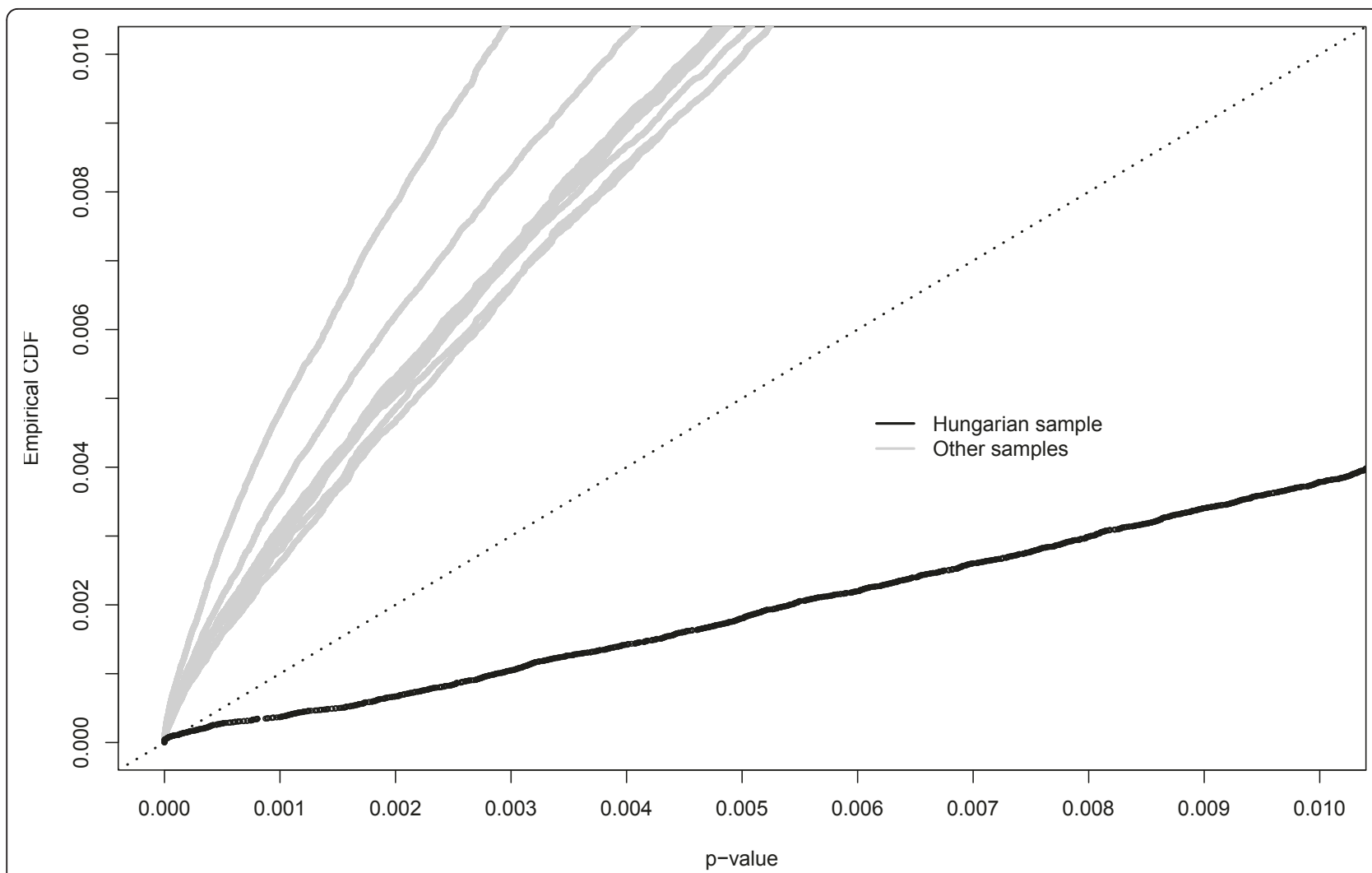

Figure 1 Empirical distributions of the $\boldsymbol{p}$-values for Thalassemia's analysis. Empirical distributions of the $p$-values of Fisher test based on $\mathbf{D}$ and on other balanced subsamples where controls have been assigned randomly to cases.

variability than other subsamples obtained ignoring the genealogy. Finally, using $\mathcal{R F}$ with the genealogical tree we are more likely to trace back the position of the mutation point causing Beta-Thalassemia.

\section{Application to Asthma}

Over a data set of 208 genotyped subjects underwent clinical and instrumental examination, we dispose of a total of

Table 1 Comparison of prediction errors for Thalassemia's analysis

\begin{tabular}{lccc}
\hline Sample Id. & First SNPs & Error (\%) & Distance (in Kb) \\
\hline Hungarian & rs7124435 & 12 & 394 \\
Random 1 & rs12271916 & 32 & 581 \\
Random 2 & rs12271916 & 32 & 581 \\
Random 3 & rs16932946 & 33 & 278 \\
Random 4 & rs12271916 & 32 & 581 \\
Random 5 & rs12271916 & 32 & 581 \\
Random 6 & rs12271916 & 32 & 581 \\
Random 7 & rs1378738 & 32 & 316 \\
Random 8 & rs12271916 & 32 & 581 \\
Random 9 & rs12271916 & 32 & 581 \\
\hline
\end{tabular}

Prediction error and distance from the HBB gene for the first important SNP for all analyzed samples in Thalassemia's analysis.
$I=57$ asthmatic cases and $J=151$ non asthmatic potential controls. For each subject we measured breath nitric oxide, forced expiratory volume (FEV1) and blood IgE levels. Moreover a standardized epidemiological questionnaire was administered to all participants to the study. The affection status has been then assessed by a clinician according to the GINA (Global INitiative for Asthma) guidelines. The total amount of considered SNPs is $P=$ 500192, each of them with two or three configurations. For each individual sex, age, smoking habits and degree of physical and sport activity is known. The latter has been classified in three categories: scarce, moderate and intense. We also dispose of 561 further subjects who did not undergo a clinical assessment for asthma but did participate to the epidemiological survey reporting their health status for whom genotypes are available. This sample will be used for validation purposes by looking at the association of the asthma status, predicted by the $\mathcal{R F}$ method, with asthma status self-reported by subjects.

The genealogical tree has been summarized into the matrix of kinship coefficients, $\mathbf{K}^{*}$, showed in Figure 2. High coefficients are symbolized by bright rectangles that also indicate highly related individuals which constitute the subsample, $\mathbf{D}$, used to estimate $\widehat{F}(\mathbf{x})$. 


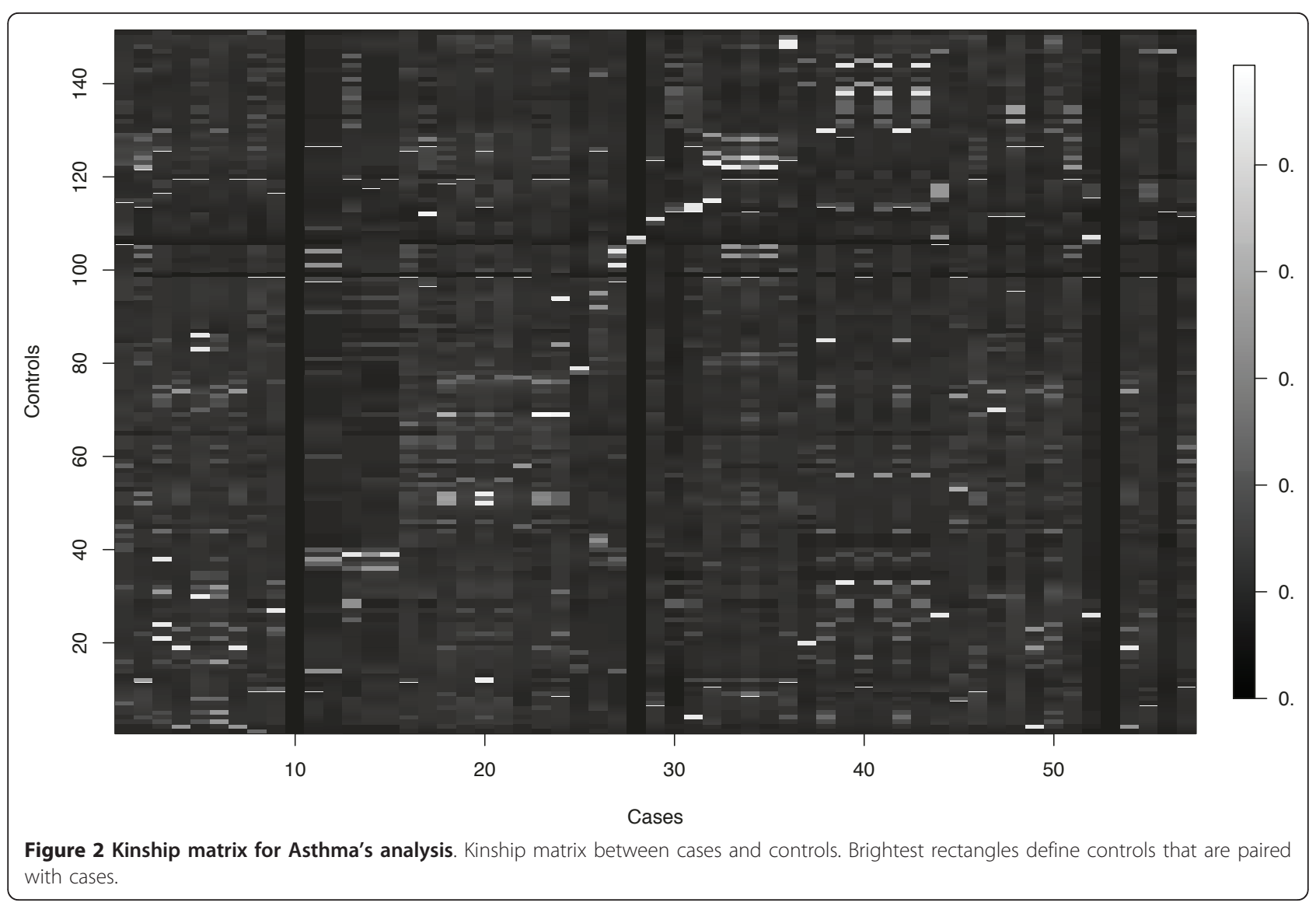

After a first screening with the Bagging approach, from $P$ variables, we end up with a list of $P^{\prime}=200$ variables. Such variables are all SNPs, while environmental variables do not play almost any role in predicting asthma if compared with these 200 SNPs.

From Figure 3 we can see how the estimated prediction error, $\Lambda$, decreases when using $\mathcal{R} \mathcal{F}$ with the first most important SNPs, in particular with the first 100 SNPs, used for the final model, the estimated value of $\Lambda$ is about $15 \%$. It is important to stress that $15 \%$ is almost three times less than $50 \%$, which is the initial classification error of a balanced sample.

We further validate the estimated prediction model $\widehat{F}(x)$, with the first 100 most important SNPs, on the 561 genotyped individuals, not including in the previous $I+J=208$ subjects, by looking at the association of the predicted asthma state, $\widehat{F}(\mathbf{x})$, with the self-reported diagnosis. It is worth noting that comparing the clinically assessed individuals to the self-reporting ones we observed an agreement on the $90.9 \%$ of subjects. The association between $\widehat{F}(\mathbf{x})$ and the anamnestic data is highly significative ( $p$-value $\left.\approx 10^{-6}\right)$. Among the 68 asthmatic, 52 have been correctly classified by $\widehat{F}(\mathbf{x})$, while the 493 non-asthmatic 271 have been correctly classified. Essentially we have that individuals predicted to be healthy and having an healthy anamnesi are more than the number expected under the hypothesis of independence between anamnesi and the predicted asthma status, $\widehat{F}(\mathbf{x})$. The same, but in the reverse, can be noted for the frequency of those classified as asthmatic which also exhibit an asthmatic anamnesi more than that expected under the hypothesis of independence.

This evidence may suggest that the 100 SNPs, which we claim to be important in classification, are functionally related to the biological processes underling common asthma. However, these results are speculatory and would need further validation. The first ten most important SNPs found with $\mathcal{R} \mathcal{F}$ are reported in Table 2. The most important SNP is inside $P$ KNOX2, a transcription factor which has been shown to be up-regulated in vascular endothelial cells stimulated by interleukin 4 [15], which is one of the most replicated gene in asthma [16]. This gene could act on asthma downstream the IL4 taking effect on the insurgence of the pathology. The second SNP is inside Hs.462615, for which almost no information is available, except that it is a sequence found expressed in pulmonary artery endothelial cells (human UniGene). The third most important SNP is 


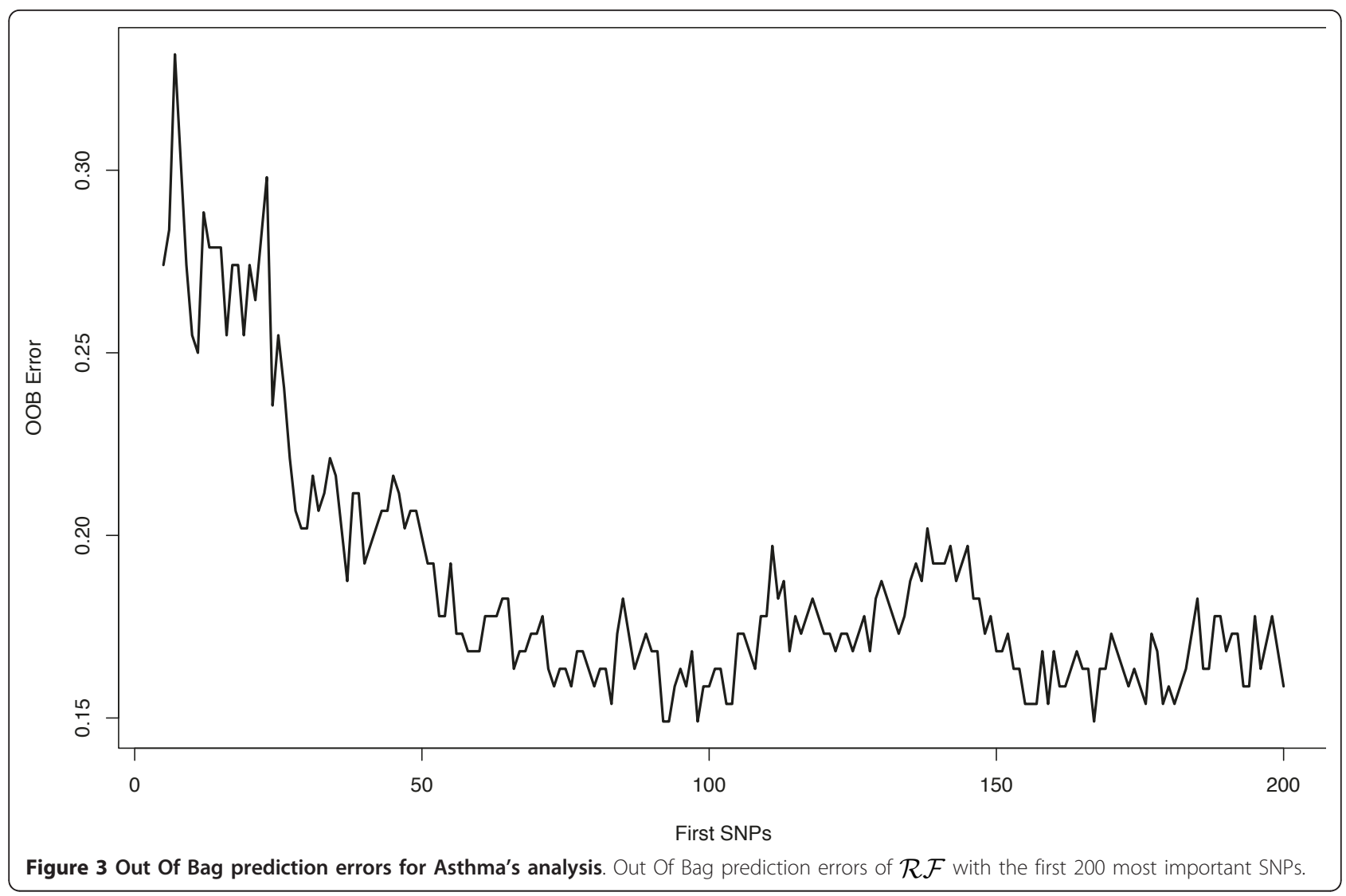

inside gene ENST00000261401 that codifies the Coronin of type 1 . Coronin of type 1 binds the actin protein which has been associated to non allergic asthma in rats according to [17]. We further applied the Mixed Effect (ME) model discussed in [18-20] to all 208 available subjects and we end up with a list of the 10 most important SNPs (Table 3) which differ from those obtained with the $\widehat{F}(\mathbf{x})$ as they are located on different genomic regions. The main problem with ME model is that we cannot regress the binomial response $Y$ against all $P$ SNPs, but instead we have to regress each SNP separately, thus losing interactions that may exists between SNPs.

\section{Simulation study}

In this section we show the results of a small simulation study with the main objective of studying differences among Receiver Operating Characteristic (ROC) curves when considering and ignoring the kinship among individuals. Beside $\mathcal{R} \mathcal{F}$ we consider: the $\mathrm{BH}$ method for
Table 2 Asthma's analysis with $\mathcal{R} \mathcal{F}$ model

\begin{tabular}{ccccc}
\hline Order & SNPs & Gene & Chrom. & Chrom. Pos. \\
\hline 1 & rs12273350 & PKNOX2 & 11 & 125241046 \\
2 & rs1254673 & no & 10 & 44473793 \\
3 & rs10861957 & CORO1C & 12 & 109056377 \\
4 & rs3105377 & no & 7 & 68837002 \\
5 & rs10004892 & no & 4 & 189872850 \\
6 & rs434949 & no & 11 & 29602274 \\
7 & rs9524111 & GPC6 & 13 & 94169901 \\
8 & rs1918215 & no & 12 & 77750550 \\
9 & rs7958647 & no & 12 & 77747825 \\
10 & rs10746129 & CORO1C & 12 & 109091089
\end{tabular}

First 10 most important SNPs for Asthma's analysis and their corresponding location inside chromosomes.
Table 3 Asthma's analysis with Mixed Effect model

\begin{tabular}{ccccc}
\hline Order & SNPs & Gene & Chrom. & Chrom. Pos. \\
\hline 1 & rs2505506 & CSGALNACT2 & 10 & 43645854 \\
2 & rs2813829 & no & 7 & 24253168 \\
3 & rs739854 & no & 7 & 24257317 \\
4 & rs7559302 & PARD3B & 2 & 205951603 \\
5 & rs16218 & no & 7 & 24257655 \\
6 & rs16212 & no & 7 & 24264661 \\
7 & rs2642265 & no & 7 & 24253062 \\
8 & rs16997879 & no & 20 & 51909584 \\
9 & rs16217 & no & 7 & 24257706 \\
10 & rs94967 & no & 21 & 41195307 \\
\hline
\end{tabular}

First 10 most important SNPs for Asthma's analysis using all samples $n=208$ with Mixed Effect model. 
multiple testing [13,14], the Q-values described in [21], the Efron's procedure in [22] and the ME model also applied for the asthma. It is worth to mention that the comparison of different methods is very difficult and not clear, because each method is specific and tailed for certain desirable features. For instance, among the methods that control an error rate for multiple testing, different methods claim to control the FDR. However, they indeed control different definition of the FDR, because the BH method [13] controls a FDR whose definition differs from the positive FDR of the $\mathrm{Q}$-values in [21] and the local FDR in [22]. We left to the reader to look at [23] for a general discussion on comparison among these methods. Further more, the three mentioned methods differ from the $\mathcal{R F}$ in that they do not explicitly estimate the $F(\mathbf{x})$ model, while they just assume that there exists one that belongs to a certain class of models as the one specified in [14] for the $\mathrm{BH}$ procedure. Moreover, the comparison between the importance of a predictor with $\mathcal{R F}$ and the corresponding significance of a regression coefficient is challenging because they belongs to different metrics as illustrated in [24]. In particular, comparison against the GEE logistic regression discussed in [25] and [26] is left as further interesting work. However, the main advantage of $\mathcal{R F}$, against regression models, is that nonlinearities and interactions can be learned from the data without any need to be specified beforehand.

Although an extensive comparison of the $\mathcal{R F}$ with other classification methods is beyond the scope of the paper (on this purposes see, for instance, [27]), we consider a small simulation study were we compare the area under the ROC curve of $\mathcal{R F}$ and the three mentioned multiple testing approaches with the primary intention of assessing the differences under two scenarios: when the kinship is taken into account, as the case of this paper, and when it is ignored.

Here we simply assume that each method under comparison is able to sort $P$ variables according to their level of association, whether or not this is significative. Considering $P$ cut points on each list of important variables, we are able to build a ROC curve and measure the corresponding area.

In this simulation study consider $N=50$ subjects and $P=1000$ SNPs randomly drawn from the 805 subjects and the 429378 SNPs available from the data set of Thalassemia study where kinship between subjects is known. We assume that only a subset of $p=5$ SNPs are related to $Y$ by a random configuration of the 5 SNPs. This configuration changes for every one of the 1000 draws and cases are assigned to the 10 subjects where $\operatorname{Pr}(Y=$ $\left.1 \mid x_{1}, \ldots, x_{p}\right)$ is the highest. Probabilities are obtained according to the logit transformation of the number of the $p$ SNPs that satisfy the random configuration. In this way we always have $I=10$ cases and 40 controls to be used to form a sample with $2 I=20$ subjects. Boxplots in Figure 4 illustrate the sample distribution of the area under the ROC curve for the 1000 replications. For each method under comparison we have two sets of box-plots: the grey one where $N-I$ controls are randomly assigned to cases $I$ and the other where they are assigned according to the Hungarian method. Although the limitations of the simulation study, results reflect what found with empirical evidence: $\mathcal{R F}$ tends to perform better with respect to other methods. It improves when the subjects are selected according to the Hungarian method because of the reduced amount of noise on $P$ - $p$ non important SNPs. The local false discovery rate and the Q-values perform worse than $\mathcal{R F}$ and the $\mathrm{BH}$ method because they are adaptive methods in the sense that they make an estimation of the null distribution of the $p$-values, while the $\mathrm{BH}$ method is not adaptive (for more details see [28]). Estimating the null distribution may be problematic because the sample size is quite small and the observed $p$-values from the Fisher test either concentrate on 0 or on 1 . This result validates the choice of the comparison between $\mathcal{R F}$ and Fisher test made for the application to Thalassemia illustrated in the above section. ME model performs similarly to the BH method. For larger samples, namely $N>100$, all methods performs equally in terms of ROC curves.

Another message conveyed by Figure 4 is that improvement of methods, under the Hungarian sample, it is not specific to the $\mathcal{R} \mathcal{F}$, but it may be also substantial for other genome-wide scanning methods.

\section{Conclusions}

We propose a strategy analysis that leads to a statistical procedure which allows us to estimate the status of genetic disease, based on the omic profile, and also provides more insights on the biological background of the disease.

Differently from usual methods, we make a multipoint analysis where interactions among all genes are considered and estimated. The method also provides a way to reduce spurious genetic variability by introducing the genealogical information into the analysis through a suitable experimental design.

We think that this strategy may be a reference one for analyzing data from genetic isolated populations, where the degree of relativeness is known.

\section{Methods}

The two critical points of genetic association studies of isolated populations with known genealogy are addressed here. In particular, dependency among subjects is accounted in the following section with a 


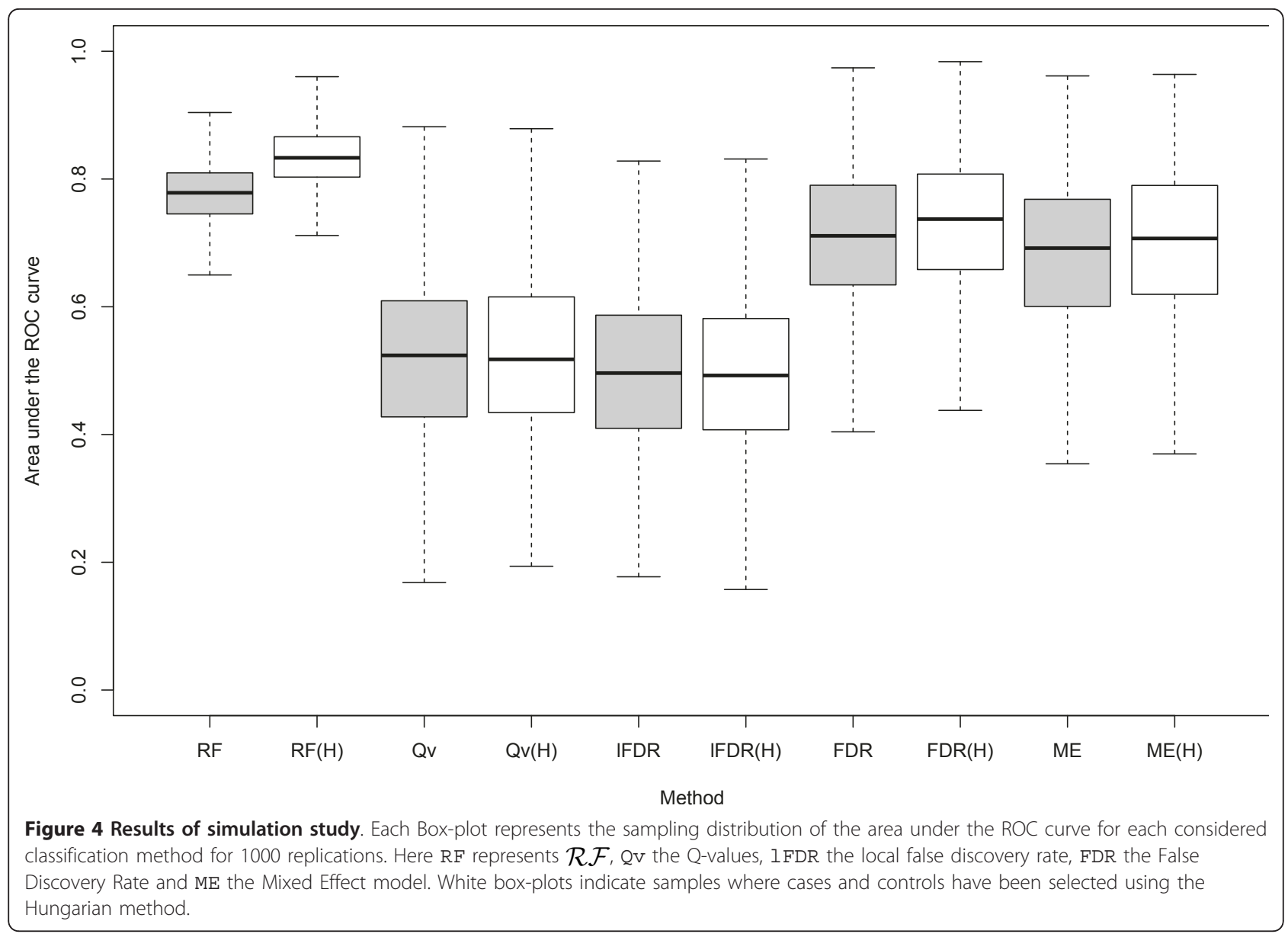

suitable experimental design, based on Hungarian method. The prediction model $F(\mathbf{x})$ and its estimation, based on Random Forest $(\mathcal{R F})$, is described in the Model Section.

The Hungarian method has been implemented in $R$ (library clue, http://www.r-project.org) while for the $\mathcal{R} \mathcal{F}$ we recurred to the open source project named PArallel Random Forest (PARF, http://code.google.com/p/parf). The latter is implemented in Fortran 95 and compiled with Open MPI libraries http://www.open-mpi.org.

\section{An experimental design that accounts for genealogy} Estimation of model $F(\mathbf{x}), \widehat{F}(\mathbf{x})$, is based on a subset, $\mathbf{D}$, of the available random sample of size $N, \mathbf{D}^{\prime} \equiv\left\{y_{n}, \mathbf{x}_{n}\right\}_{n=1}^{N}$. Variable $y_{n}$ represents the disease status of the $n$-th subject, where $y_{n}=1$ if the subject $n$ has been diagnosed with the disease and $y_{n}=0$ otherwise. Suppose to have $I=\sum_{n=1}^{N} y_{n}<N$ cases and $J=N-I$ controls, where cases and controls have been labeled according to the medical diagnosis of the disease. Vector $\mathbf{x}_{n}$ is of size $P$ and contains SNPs configurations and covariates that resemble habits and individual characteristics of subject $n$. The vector $\mathbf{x}_{n}$ defines the omic profile of subject $n$ which we use to predict the disease status $Y$.

We are interested in using a balanced sample of size $2 I$ where the genetic variability, exogenous to the disease, is reduced. The idea to obtain such sample consists in accounting for relatedness among individuals of different disease status, which we can do taking advantage of the availability of the entire genealogy of the investigated isolated population. For instance, if we consider two individuals, one affected and one not, we are more likely to identify disease predisposing variants if they were brothers rather than if they were unrelated.

The genetic variability, between two individuals, increases with the number of meiotic steps that separate them into the genealogical tree. Such number define relatedness among two individuals. The most common used measure of relatedness between two individuals $i$ and $j$, is the kinship coefficient [11], $k_{i j}$, which represents the probability that two genes, sampled at random from each individual, are identical because inherited from the same ancestor (IBD). For instance $k_{i j}=1 / 4$ if $i$ is the parent of individual $j$, while $k_{i j}=0$ zero if $i$ and $j$ are not related. 
Let $\mathbf{K}_{I \times J}^{\prime}$ be the matrix of kinship indexes between cases and controls, whose generic entry is $k_{i j}$, the kinship index between case $i, i=1, \ldots, I$ and control $j, j=$ $1, \ldots, J$. Finding a subset of size I of controls most related to the $I$ cases corresponds to find a sub-matrix $\mathbf{K}_{I \times I}$ of $\mathbf{K}^{\prime}$ with the $I$ columns made by a set of indexes $\mathcal{J} \equiv\left\{j_{i}^{*}\right\}_{i=1}^{I}$ such that $\sum_{i=1}^{I} k_{i j_{i}^{*}}$ is maximized over all possible sets of indexes $\mathcal{J}$.

This is an assignment problem, which is typical of combinatorial optimization area, and that has been solved with the well known Hungarian algorithm. This method was, for many years, attributed to H. Kuhn who developed and published it in [9]. However, in 2006, it was discovered that Carl Gustav Jacobi solved the assignment problem in the 19th century, and published posthumously in 1890 in Latin [10].

In the sequel, we assume that the prediction model $F$ $(\mathbf{x})$ is estimated on the sub-sample $\mathbf{D} \subset \mathbf{D}^{\prime}$ where controls correspond to the columns of $\mathbf{K}$.

The design of such experiment provides a balanced case/control study with $2 I$ individuals, where spurious genetic variability is supposed to have been reduced.

\section{Model}

In order to obtain the prediction model, $F(\mathbf{x})$, for a certain omic profile, $\mathbf{x}$, we propose a procedure based on Random Forest $(\mathcal{R F})$ described in [29]. $\mathcal{R} \mathcal{F}$ is a non parametric regression model and its use in genetic association studies grows only in the very recent years. In particular, [27] and [30] use it with expression data, where the considered values of $P$ are of order of a few thousands and the observations are assumed independent. On the contrary, most genetic association studies consider only one genetic variant at time and rarely take into account the interactions between them [12]. Other papers, based on multiple testing [23,31], take into account interactions among genes, but they do not explicitly model such dependency as done in this paper.

We briefly describe $\mathcal{R F}$ with particular emphasis to those variations of the algorithm proposed in this work. $\mathcal{R F}$ is an additive model given by the ensemble of $M=$ $10^{6}$ non-parametric classifiers. In our case these are classification trees, $\left\{h_{m}\left(\mathbf{a}_{m}\right) \in\{0,1\}\right\}_{m=1}^{M}$, with the usual Bernoulli deviance, where $\mathbf{a}_{m}$ denotes the regions of the space spanned by the $P$ variables. For more details see [32]. Each $h_{m}$ is based on a random sample of size $p=\sqrt{P}$ of the $P$ variables and a corresponding random sample of size $[I / 2]$ individuals. This sample is called the in bag sample, denoted by $\mathbf{D}_{i b}$ in order to be distinguished by the out of bag (OOB) sample denoted with $\mathbf{D}_{o o b}=\mathbf{D} \backslash \mathbf{D}_{i b}$. Both sets, $\mathbf{D}_{o o b}, \mathbf{D}_{i b}$ represent the $m$-th random partition of $\mathbf{D}$ and the classification error of $h_{m}$ is always estimated with $\mathbf{D}_{o o b}$ sample of size $I$ - [I/2].
This is done in order to avoid overoptimistic prediction errors. The parameter $\mathbf{a}_{m}$ contains splitting variables that are supposed to be the part of the omic profile related to the disease according to the $m$-th tree. The prediction model is given by the mean prediction of the $M$ trees:

$$
\widehat{F}(\mathbf{x})=\frac{1}{M} \sum_{m=1}^{M} h\left(\mathbf{x} ; \mathbf{a}_{m}\right) .
$$

In this application, each $h_{m}\left(\mathbf{a}_{m}\right)$ estimates part of the complex relation between the omic profile and the disease, while the whole estimation of such complex model is given by $\widehat{F}(\mathbf{x})$.

If $\widehat{F}(\mathbf{x})>0.5$ then the profile $\mathbf{x}$ belongs to an ill individual otherwise to a healthy one. Note that $\widehat{F}(\mathbf{x})>0.5$ is also the Bayes rule under prior probabilities $\operatorname{Pr}(Y=1)$ $=\operatorname{Pr}(Y=0)=0.5$. The threshold, 0.5, may be changed according to different prior probability for the disease of interest. Such prior may be suggested by the prevalence of the disease in the population of interest.

The validation of $\widehat{F}(\mathbf{x})$ is made by means of the prediction error for the outcome $Y$ of the $m$-th tree, $\lambda_{m}$ (see [29]), estimated over $\mathbf{D}_{\text {oob }}$. Let $\Lambda_{M}$ be the expected prediction error of $M$ trees, where expectation is calculated for the joint distribution of $(Y, \mathbf{x})$. According to Theorems 1.2 and 2.3 in [29], as $M \rightarrow \infty$,

$$
\Lambda_{M} \rightarrow \Lambda \leq \rho\left(1-s^{2}\right) / s^{2}>0, \text { almost surely, }
$$

where $s=1-E_{(Y, \mathbf{X})}\left(\lambda_{m}\right)$, is the classifier's strength, and $\rho$ is the correlation among classifiers, also known as classifier's diversity. Under conditions of Theorems 1.2 and 2.3, $\mathcal{R F}$ does not over-fit if we arbitrary increase $M$. A more general discussion on consistency of $\mathcal{R F}$ and other classification methods can be found in [33].

Using $\mathcal{R} \mathcal{F}$ we obtain a non-parametric regression model that we can use to make prediction and variable (SNP) selection at the same time. Variable selection is performed by means of each $h_{m}$, because $\mathbf{a}_{m}$ is obtained by using only those variables that assure the largest decrease of the Bernoulli deviance.

Therefore, for each of the $P$ variables we can consider its importance according to the total amount of decreasing of the Bernoulli deviance induced in all $M$ trees, say $\left\{\eta_{j}\right\}_{1}^{P}$. We can then produce a rank of the $P$ variables by sorting them into a decreasing order according to their respective $\eta_{j}$. The interpretation of the set $\left\{\eta_{j}\right\}_{1}^{P}$ is that each genetic variant contributes to the complex genetic model, but some of them are more important than others. The greater the $\eta_{j}$ the more likely is that variable (SNP) $j$ is a risk or protective factor for the disease. 
Another advantage of $\mathcal{R F}$ is that it is highly parallelizable, in fact we implemented it for running in a cluster of CPUs, but it could also be implemented to run on GPUs (Global Processor Units, gpu . sourceforge. net). This approach makes $\mathcal{R} \mathcal{F}$ a suitable approach for association studies with high numbers of combination of genetic variants that interact one with the other. Moreover, the simulation study in [34] showed that $\mathcal{R F}$ is able to detect true associations under a purely interactive model.

\section{A Bagging strategy for genome-wide association studies}

Alleles of a specific genetic variant are usually associated to the alleles of the neighboring variants due to the lack of recombination between them. This effect is referred to as Linkage Disequilibrium (LD) [35]. LD usually decreases as distance between markers increases. Translating this into statistical terms we have that variables of the omic profile are not independent, but they rather show a clumpy dependence as illustrated in Figure 5(a). Moreover, as the whole genome is not accessible, we exploit LD in order to know the configuration of the variables surrounding the one we have genotyped. This means that, although we do not observe mutations responsible for the disease we can still catch this information through LD as illustrated in Figure 5(b).

Association studies aim at discovering causal variants that are responsible for the disease and these can be located by those SNPs that are nearest to the causal variant. In order to have a good prediction model, we

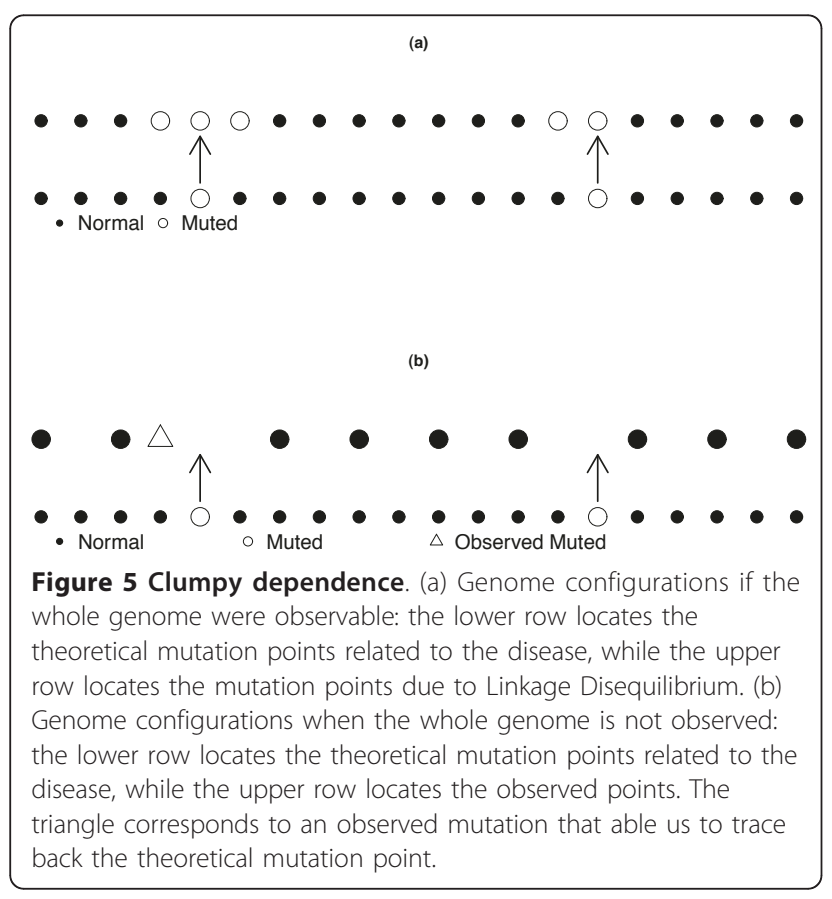

should exclude all SNPs that, although having some predictive power on the outcome $Y$, are far from the causal variant and they are essentially false positives. Statistically speaking, we have to make a screening among all SNPs before proceeding to estimate the final model $F$ $(\mathbf{x})$. After the screening phase we construct the final model, by selecting the most associated SNPs. These two phases are related to the screening and cleaning procedures advocated in [36]. The work of [36] shows the consistency of this strategy in the context of classical regression models.

For the screening procedure we noted that each $h_{m}$ makes the subset of the $p$ variables randomly chosen to compete, by using, as splitting variables, only those that are most associated with $Y$ (i.e. the highest decrease in the Bernoulli deviance). Therefore, only SNPs most related to I are chosen in each tree. If $p \ll P$ then each $\mathbf{a}_{m}$ is estimated by the competition between only $p$ variables. For this reason it is likely that in $M$ trees there would be many different SNPs that are selected to form the final $\widehat{F}(\mathbf{x})$. This approach, which is the usual one in $\mathcal{R F}$, may produce a final model in which many SNPs are included just because each $\mathbf{a}_{m}$ refers to different sets of predictors. In order to avoid such false positives, we start by running $\mathcal{R F}$ in Bagging mode, that is with $p=$ $P$, implying that all $\mathbf{D}_{i b}$ in $M$ trees differ only for individuals but not for variables. The set $\left\{\eta_{j}\right\}_{1}^{P}$, corresponding to the bagging, allows us to select those SNPs that are most related to $Y$ by eliminating all variables with $\eta_{j}=$ 0 . We actually expect many variables with zero importance, because such variables have never been used in any of the $M$ trees. We then repeat the bagging procedure on the remaining set of variables until all $\eta_{j}>0$. This screening phase eliminates all those false positives due only to LD while retaining those SNPs that bear important information. For example, in the case of an association to an haplotype instead of a single SNP on a gene, we expect that only SNPs important to the haplotype would be retained.

We end up, with the screening phase, with a set of $P^{\prime}$ variables and the corresponding model $\widehat{F}(\mathbf{x})$ has a high $\Lambda$ just because trees only differs for individuals and not for the used variables, therefore its correlation $\rho \rightarrow 1$. In order to make $\rho \rightarrow 0$, we run again $\mathcal{R F}$ with the k most important variables among the selected $P^{\prime}$ using the rule suggested in [29], that is $p=[\sqrt{k}]$ for $k=1, \ldots$, $\mathrm{P}^{\prime}$, and $[\sqrt{k}]$ denotes the nearest smallest integer to $\sqrt{k}$. We start estimating $\widehat{F}(\mathbf{x})$ with all the elements in the list according to $\left\{\eta_{j}\right\}_{1}^{P^{\prime}}$ and we subsequently remove one variable at a time until the estimated value of $\Lambda$ starts to increase. The final list of variable (SNPs) is the one obtained by running the RF on the increasing sets of 
most important variables obtained with Bagging. This set is the smallest one that produces the lowest prediction error. This is also the set of variables supposed to be most associated with $Y$ and that produces the smallest classification error with $\mathcal{R F}$.

\section{Remarks}

As final remarks, we would like to comment some aspects of the methods we used:

- all covariates in $\mathbf{X}$ have almost the same number of categories, 2 or 3, both for SNPs and environmental variables. This is important because it is known that if the number of categories are very different among predictors, or if continuous covariates are mixed with categorical ones, then the latter would result to be most important than categorical variables with a small number of categories. Essentially, there would be a BIAS in assessing variable importance with $\mathcal{R F}$ as noted in [37]. The BIAS is due to the fact that a variable with more levels or a continuous variable is more likely to be a splitting variable with respect to another discrete variable with a lower number of levels. This could affect situations in which there variables with different number of levels, however it is not the case in our applications. Finally, it would be interesting to compare variable importance by looking at their distance from the root node of the tree as recently proposed in [38] for survival analysis. At the moment, this is beyond the scope of the paper;

- the huge number of predictors, say $P \approx 500000$ (but $P \approx 10^{6}$ is on the wing), requires parallel computing and huge amount of storage and fast access memory. Therefore, it is difficult to manage the kind of analysis, here discussed, by classical regression methods which usually require to calculate $\left(\mathbf{X}^{T} \mathbf{X}\right)^{-1}$. Other alternatives to $\mathcal{R} \mathcal{F}$, based on ensemble methods, could be the gradient boosting algorithm [39] or its stochastic version [40]. From a Bayesian perspective, we note that the Bayesian Additive Regression Trees (BART), proposed in [41], could be an alternative because it allows to draw explicit causal relations among genetic variants and disease status. In our study, we rather draw simple associations. Unfortunately, BART is much more difficult to be parallelize than $\mathcal{R F}$ and the computational e ort would be not affordable with a standard machine used for the present study;

- the kinship coefficient is a first attempt to summarize the genealogical tree and some information may be lost. To avoid this, it would be possible to directly use the genealogy in the construction of each classification tree $h_{m}$. This would be in line with the Breiman's prescription which requires that all aspects of growing a random forest take into account the outcome.

- the proposed design consisting in selecting the controls most inbred with cases could be potentially applied to other methods for association studies, beginning with the very simple Fisher-test as done in the simulation study of Figure 1 for the Thalassemia and also in the simulation study where alternative approaches to the $\mathcal{R} \mathcal{F}$ are compared. At the moment, we have no evidence for stating that the combination of the design and Random Forest has unique advantages; - rare causal variants, which have a very small effect, can be a problem for RF because each of the $M$ trees are grown until the subjects in the final node are less than a specified quantity which is 2 subjects in the present study. Therefore, if there are rare causal variants and they have an effect so small that less than 2 individuals are affected, these cannot be detected with the proposed $\mathcal{R F}$ approach. However, such variants are, in general, very difficult to be detected.

\section{Acknowledgements}

This work was supported by the following institutions:

- Italian Ministry of Education, University and Research, grants no. 2008275CMW-002 and no. 5571/DASPAR/2002;

- The visiting professor program of Regione Autonoma della Sardegna (Italy); - Ministerio de Ciencias e Imnovaci'on of Spain, grant no. MTM2010-19528;

- Comunidad Autonoma de Madrid of Spain, grant no. CM. S2009/esp-1594; - Project ICT SIAI 101 "Creating a center of excellence in bio-computing technologies applied to personalized medicine" Sardegna Ricerche, Sardinia Region, Ministry of Economy and Finance.

\section{Author details}

'Department of Mathematics and Informatics, University of Cagliari, Cagliari, Italy. ${ }^{2}$ Department of Statistics and O. R., Rey Juan Carlos University, Mostoles, Madrid, Spain. Institute of Population Genetics CNR, Alghero (SS), Italy. ${ }^{4}$ Shardna Live Science, Loc. Piscina Manna, Pula (CA), Italy. Institute of Molecular Genetics CNR, Pavia, Italy. ${ }^{6}$ Department of Medical Science, University of Cagliari, Cagliari, Italy. ${ }^{7}$ Medical Genetics, IRCCS-Burlo Garofolo, University of Trieste, Trieste, Italy.

\section{Authors' contributions}

SC developed and implemented the statistical method. MEC and FB contributed to the development of the statistical method. NP contributed to development of the method, implemented the method in R and run the RF analysis. LC contributed to the assessment of the obtained results and the construction and management of the data used in both analyses. IP and AS performed the genotyping. GB contributed to the writing. SG clinically supported this research. MP supervised the research. All authors read and approved the final manuscript.

\section{Competing interests}

The authors declare that they have no competing interests.

Received: 16 March 2011 Accepted: 18 July 2011

Published: 18 July 2011

\section{References}

1. Balding D: A tutorial on statistical methods for population association studies. Nat Rev Genet 2006, 7:781-191. 
2. Fridley BL, Serie D, Jenkins G, White K, Bamlet W, Potter JD, Goode EL: Bayesian mixture models for the incorporation of prior knowledge to inform genetic association studies. Genet Epidemiol 2010, 34(5):418-26.

3. Wasserman L: All of Nonparametric Statistics Springer; 2006.

4. Andersson T, Alfredsson L, Källberg H, Zdravkovic S, Ahlbom A: Calculating measures of biological interaction. Eur J Epidemiol 2005, 20(7):575-579.

5. Trecartin RF, Liebhaber SA, Chang JC, Lee KY, Kan YW, Furbetta M, Angius A, Cao A: beta zero thalassemia in Sardinia is caused by a nonsense mutation. J Clin Invest 1981, 68(4):1012-1017.

6. Rosatelli C, Leoni G, Tuveri T, et al: Heterozygous Beta-thalassemia: relationship between the hematological phenotype and the type of Beta-thalassemia mutation. American Journal of Hematology 1989, 39.

7. Rosatelli MC, Dozy A, Faà V, Meloni A, Sardu R, Saba L, Kan YW, Cao A: Molecular characterization of beta-thalassemia in the Sardinian population. Am J Hum Genet 1992, 50(2):422-6.

8. Cao A, Congiu R, Sollaino MC, Desogus MF, Demartis FR, Loi D, Cau M, Galanello R: Thalassaemia and glucose-6-phosphate dehydrogenase screening in 13- to 14-year-old students of the Sardinian population: preliminary findings. Community Genet 2008, 11(3):121-8.

9. Kuhn HW: The Hungarian Method for the Assignment Problem. Naval Research Logistics 1955, 2:83-97.

10. Calmet J, Ollivier F: Editors' foreword [Special issue: Jacobi's legacy]. Appl Algebra Engrg Comm Comput 2009, 20:1-4.

11. Lange K: Mathematical and statistical methods for genetic analysis SpringerVerlag, New York; 1997.

12. Balding DJ: A tutorial on statistical methods for population association studies. Nat Rev Genet 2006, 7(10):781-791.

13. Benjamini $Y$, Hochberg Y: Controlling the False Discovery Rate: a Practical and Powerful Approach to Multiple Testing. J Roy Stat Soc B 1995, 57:289-300.

14. Benjamini Y, Yekutieli D: The control of the False Discovery Rate in multiple testing under dependence. Ann Stat 2001, 29:1165-1188.

15. Lee YW, Eum SY, Chen KC, Hennig B, Toborek M: Gene expression profile in interleukin-4-stimulated human vascular endothelial cells. Mol Med 2004, 10(1-6):19-27.

16. Weiss ST, Raby BA, Rogers A: Asthma genetics and genomics 2009. Curr Opin Genet Dev 2009, 19(3):279-282.

17. Almon RR, Yang E, Lai W, Androulakis PI, DuBois CD, Jusko WC: Circadian Variations in Rat Liver Gene Expression: Relationships to Drug Actions. The Journal of Pharmacology and Experimental Therapeutics 2008, 326:700-726.

18. Thompson EA, Shaw RG: Pedigree analysis for quantitative traits: variance components without matrix inversion. Biometrics 1990, 46(2):399-413.

19. Chen WM, Abecasis GR: Family-based association tests for genomewide association scans. Am J Hum Genet 2007, 81(5):913-926.

20. Aulchenko YS, de Koning DJ, Haley C: Genomewide rapid association using mixed model and regression: a fast and simple method for genomewide pedigree-based quantitative trait loci association analysis. Genetics 2007, 177:577-585.

21. Storey JD: The Positive False Discovery Rate: A Bayesian Interpretation and the Q-value. Ann Stat 2003, 31:2013-2035.

22. Efron B: Size, Power and False Discovery Rates. Ann Statist 2007, 35(4):1351-1377.

23. Farcomeni A: A review of modern multiple hypothesis testing, with particular attention to the false discovery proportion. Stat Methods Med Res 2008, 17(4):347-388.

24. Grömping U: Variable Importance Assessment in Regression: Linear Regression versus Random Forest. The American Statistician 2009, 63(4):308-319.

25. Hancock DB, Martin ER, Li YJ, Scott WK: Methods for interaction analyses using family-based case-control data: conditional logistic regression versus generalized estimating equations. Genet Epidemiol 2007, 31(8):883-93.

26. Chen MH, Yang Q: GWAF: an R package for genome-wide association analyses with family data. Bioinformatics 2010, 26(4):580-1.

27. Díaz-Uriarte $R$, de Andrés SA: Gene selection and classification of microarray data using random forest. BMC Bioinformatics 2006, 7(3):11-22.

28. Genovese C, Wasserman L: Operating characteristics and extensions of the false discovery rate procedure. J Roy Stat Soc B 2002, 64:499-518.

29. Breiman L: Random forests. Machine Learning 2001, 45:5-32.
30. de Edelenyi FS, Goumidi L, Bertrais S, Phillips C, Macmanus R, Roche H, Planells R, Lairon D: Prediction of the metabolic syndrome status based on dietary and genetic parameters, using Random Forest. Genes Nutr 2008, 3:173-6.

31. Cabras S: A note on multiple testing for composite null hypotheses. Journal of Statistical Planning and Inference 2010, 140.

32. Hastie T, Tibshirani R, Friedman JH: The Elements of Statistical Learning: Data Mining, Inference, and Prediction Springer-Verlag; 2001.

33. Biau G, Devroye L, Lugosi G: Consistency of random forests and other averaging classifiers. Journal of Machine Learning Research 2008, 9:2015-2033.

34. Garcl'a-Magarinos M, de Ullibarri IL, Cao R, Salas A: Evaluating the ability of tree-based methods and logistic regression for the detection of SNPSNP interaction. Ann Hum Genet 2009, 73(3):360-369.

35. Lewontin RC, Kojima K: The evolutionary dynamics of complex polymorphisms. Evolution 1960, 14(4):458-472.

36. Wasserman L, Roeder K: High Dimensional Variable Selection. Ann Stat 2009, 37(5A):2178-2201.

37. Strobl C, Boulesteix A, Zeileis A, Hothorn T: Bias in Random Forest Variable Importance Measures: Illustrations, Sources and a Solution. BMC Bioinformatics 2007, 8(25).

38. Ishwaran H, Kogalur UB, Gorodeski EZ, Minn AJ, Lauer MS: HighDimensional Variable Selection for Survival Data. Journal of the American Statistical Association 2010, 105(489):205-217.

39. Friedman JH: Greedy Function Approximation: A Gradient Boosting Machine. The Annals of Statistics 2001, 29(5):1189-1232.

40. Friedman JH: Stochastic Gradient Boosting. Computational Statistics and Data Analysis 2002, 38:367-378.

41. Chipman HA, George El, McCulloch RE: BART: Bayesian Additive Regression Trees. The Annals of Applied Statistics 2010, 4:266-298.

doi:10.1186/1471-2156-12-63

Cite this article as: Cabras et al:: A strategy analysis for genetic association studies with known inbreeding. BMC Genetics 2011 12:63.

\section{Submit your next manuscript to BioMed Central and take full advantage of:}

- Convenient online submission

- Thorough peer review

- No space constraints or color figure charges

- Immediate publication on acceptance

- Inclusion in PubMed, CAS, Scopus and Google Scholar

- Research which is freely available for redistribution

Submit your manuscript at www.biomedcentral.com/submit
C) Biomed Central 